2010-09

\title{
Stereotyping starlings are more
}

\section{'pessimistic'.}

\author{
Brilot, BO
}

http://hdl.handle.net/10026.1/2999

10.1007/s10071-010-0323-z

Anim Cogn

All content in PEARL is protected by copyright law. Author manuscripts are made available in accordance with publisher policies. Please cite only the published version using the details provided on the item record or document. In the absence of an open licence (e.g. Creative Commons), permissions for further reuse of content should be sought from the publisher or author. 


\section{Stereotyping starlings are more 'pessimistic'}

2

3

4

5

$6 \quad{ }^{1}$ Centre for Behavior and Evolution, Institute of Neuroscience, Newcastle 7 University, UK.

8

9

23 Keywords: Sturnus vulgaris; European starling; stereotypic behaviour; cognitive bias; environmental enrichment; anxiety Bar, UK.

Corresponding author: Dr. Ben Brilot, Centre for Behaviour and Evolution, Institute of Neuroscience, Newcastle University, Henry Wellcome Building for Neuroecology, Framlington Place, Newcastle upon Tyne, NE2 4HH, UK.

E-mail: ben.brilot@ncl.ac.uk

Running head: Stereotypic behaviour in starlings predicts a cognitive bias 


\section{Abstract}

27 Negative affect in humans and animals is known to cause individuals to interpret

28 ambiguous stimuli pessimistically, a phenomenon termed 'cognitive bias'. Here,

29 we used captive European starlings (Sturnus vulgaris) to test the hypothesis that

30 a reduction in environmental conditions, from enriched to non-enriched cages,

31 would engender negative affect, and hence 'pessimistic' biases. We also explored

32 whether individual differences in stereotypic behaviour (repetitive somersaulting)

33 predicted 'pessimism'. Eight birds were trained on a novel conditional

34 discrimination task with differential rewards, in which background shade (light or

35 dark) determined which of two covered dishes contained a food reward. The

36 reward was small when the background was light, but large when the background

37 was dark. We then presented background shades intermediate between those

38 trained to assess the birds' bias to choose the dish associated with the smaller

39 food reward (a 'pessimistic' judgement) when the discriminative stimulus was

40 ambiguous. Contrary to predictions, changes in the level of cage enrichment had

41 no effect on 'pessimism'. However, changes in the latency to choose and

42 probability of expressing a choice suggested that birds learnt rapidly that trials

43 with ambiguous stimuli were unreinforced. Individual differences in performance

44 of stereotypies did predict 'pessimism'. Specifically, birds that somersaulted were

45 more likely to choose the dish associated with the smaller food reward in the

46 presence of the most ambiguous discriminative stimulus. We propose that

47 somersaulting is part of a wider suite of behavioural traits indicative of a stress

48 response to captive conditions that is symptomatic of a negative affective state.

49

50 


\section{Introduction}

There is an extensive body of literature in human psychology showing that emotions can influence cognitive processes (Williams et al. 1997). For example, negative affective states such as anxiety can cause increased attention to threatening stimuli (Bar-Haim et al. 2007), and can increase the likelihood that ambiguous information will be interpreted pessimistically (Eysenck et al. 1991). These cognitive biases are sensitive both to short-term changes in anxiety (i.e. state anxiety) and stable individual differences in anxiety (i.e. trait anxiety) (BarHaim et al. 2007). Similar cognitive biases have also been shown to occur in animals whose states have been manipulated in various ways. Studies in laboratory rats (Rattus norvegicus; Harding et al. 2004; Burman et al. 2008a) and captive wild-caught European starlings (Sturnus vulgaris; Bateson and Matheson 2007; Matheson et al. 2008) have demonstrated that changes in husbandry that are likely to engender negative affective states cause 'pessimistic' biases in the animals' interpretation of ambiguous stimuli, i.e. they have an expectancy of a more negative outcome. For example, in a previous experiment we trained starlings on a go/no-go task to discriminate between white and dark grey cardboard lids associated respectively with palatable and unpalatable mealworms hidden underneath. Once the birds had learnt to flip the white lids and avoid the dark grey lids, we measured their judgement biases (a form of cognitive bias) by presenting them with ambiguous lids of intermediate shades of grey. When the birds were housed in un-enriched cages, they were more reluctant to approach and flip the ambiguous lids than when they were housed in enriched cages (Bateson and Matheson 2007). We interpreted this result as a pessimistic judgement bias in birds housed in environmental conditions known to be associated with poorer welfare. Cognitive bias tasks have been tentatively supported as a new tool for diagnosing negative affective states in captive animals (Paul et al. 2005; Mendl et al. 2009). However, as we will explain below, a number of theoretical and empirical issues remain (see also Mendl et al. 2009). Our aims in the current paper are (1) to develop a novel judgement task for measurement of cognitive bias in starlings intended to improve on previous tasks; and (2), to extend previous work in animals, by asking whether cognitive biases are correlated with individual differences in the incidence of abnormal behaviour, specifically stereotypies, that might also reflect trait anxiety. We present the background to each of these aims in more detail below. 


\section{Judgement bias tasks for animals}

88 The tasks developed so far to measure judgment biases in animals are based on

89 the original design of (Harding et al. 2004). Subjects are initially trained to

90 associate one stimulus, S+, with a reward (generally food) and another S- with

91 either a reward of lower value (e.g less food), or a punishment (e.g. white noise

92 or a noxious food item). S+ and S- are chosen to lie on a continuous stimulus

93 spectrum (e.g. a frequency range for auditory stimuli, a spectral range for visual

94 stimuli or a range of directions for spatial stimuli). To measure a cognitive bias,

95 subjects' responses to novel stimuli ('probes') that are intermediate between the

96 trained stimuli are recorded in extinction (i.e. probe trials are not reinforced,

97 avoiding any confound of reinforcement). A subject is regarded as showing a

98 pessimistic judgement bias if it demonstrates a higher probability of exhibiting

99 the response appropriate to the S- stimulus, than either that same subject in a

100 more positive affective state or when compared to control subjects in a more

101 positive state.

102 To date, the majority of published cognitive bias tasks have used a go/no-

103 go procedure (Harding et al. 2004; Bateson and Matheson 2007; Burman et al.

104 2008a). In a go/no-go task the subject is required to respond by performing a

105 behaviour (e.g. lever press) in response to $\mathrm{S}_{+}$, but to refrain from responding to

106 S-. However, interpretation of data from a go/no-go task is complicated by the

107 possibility that negative affective states are often associated with changes in

108 general activity and feeding motivation. Therefore, on a go/no-go task, it is

109 possible that subjects in a more negative affective state show a reduced

110 probability of responding because they are less motivated to exploit a signalled

111 food source, rather than because they interpret the ambiguous stimulus

112 pessimistically. Thus, go/no-go tasks could be measuring a more general

113 response bias as opposed to the assumed biased judgement of the ambiguous

114 stimulus.

115 To address the above problem with go/no-go tasks we, and others, have

116 advocated the use of choice tasks whereby subjects are required to make a

117 different active response to both S+ and S- stimuli (e.g. Matheson et al. 2008;

118 Enkel et al. 2009); see also unpublished studies cited in Mendl et al. 2009). This

119 experimental design allows the effects of a response bias and a judgement bias to

120 be distinguished: the former should result in reduced responding to all stimuli

121 whilst the latter should result in reduced responding only to ambiguous stimuli. In

122 a previous study with starlings we used an operant task in which birds were

123 required to choose (by pecking) a red or green key to classify a light stimulus as

124 S+ or S- (Matheson et al. 2008). However, this task has a number of practical 
125 limitations including the length of time taken to train the birds, the requirement

126 for moderate levels of food deprivation and the requirement to catch and handle

127 the birds daily to transfer them to the operant chambers (a potentially anxiety-

128 inducing procedure; Rich and Romero 2005). In the current paper we present a

129 novel choice task that is a modification of the simple lid-flipping task described

130 earlier (Bateson and Matheson 2007). The task was designed to be quick to train,

131 and minimally disruptive to the birds, with all training and experimental

132 procedures conducted in the home cages (c.f. Matheson et al. 2008).

133 In line with previous cognitive bias experiments, we manipulated the

134 affective state of the birds by altering the level of environmental enrichment

135 provided in their cages (Bateson and Matheson 2007; Matheson et al. 2008).

136 There are extensive data from a wide range of species showing that provision of

137 more enrichment in captive animals' cages is associated with better welfare

138 (Young 2003), and we have data from our own laboratory showing that starlings

139 in enriched cages display less abnormal behaviour (Asher 2007). We used a

140 repeated measures design involving a sequential change from environmentally

141 enriched conditions, to non-enriched conditions and then returning to enriched

142 conditions. This design delivers greater statistical power in a study involving low

143 numbers of subjects (a constraint of the intensive training required), and

144 additionally allows us to examine how starlings respond to both reduction and

145 improvement in their environmental conditions. We have previously found that

146 starlings show a greater change in cognitive bias in response to a reduction in

147 environmental conditions than to an improvement (Bateson and Matheson 2007),

148 adding to many results showing that animals' responses to a given situation

149 depend on what they have previously experienced (Flaherty 1996; Bergvall et al.

150 2007; Burman et al. 2008b).

151 We hypothesised that starlings would show more pessimistic judgement

152 biases in the non-enriched conditions compared with the enriched conditions. We

153 also hypothesised that the birds would show a greater response to the removal of

154 environmental enrichment than to its reinstatement in the final stage of the

155 experiment.

\section{Individual differences}

157 The published cognitive bias studies in animals thus far have all examined

158 whether judgement biases are sensitive to relatively short-term manipulations of

159 state (Harding et al. 2004; Bateson and Matheson 2007; Burman et al. 2008a;

160 Matheson et al. 2008). However, the literature in humans suggests that there are

161 also stable individual ('trait') differences in both affect and pessimism (Bar-Haim 
162 et al. 2007). How individual animals cope with captivity is not only a matter of

163 animal welfare, but is also of concern for the scientific validity of studies related

164 to cognition. Differences in coping ability might be reflected in an animal's

165 affective state and hence in the choices they make, regardless of the

166 experimental treatment. Repetitive, abnormal behaviour (of which stereotypy is a

167 type) is often regarded as an indicator of poor welfare since this behaviour can be

168 associated with physiological and behavioural measures of stress (Mason and

169 Rushen 2006). The evidence linking stereotypy and poor welfare is, however,

170 mixed: a review of 90 studies in a range of species found that comparing

171 between environments or regimes, those where the subjects stereotyped more

172 invariably also scored lower on additional welfare measures, but within a group of

173 animals under the same husbandry regime, $60 \%$ of studies showed that

174 performance of stereotypies was associated with better welfare whilst the

175 remainder showed the opposite (Mason and Latham 2004).

$176 \quad$ Given the above, we hypothesised that the presence of stereotyping

177 behaviour in individual starlings should reflect stable individual differences in

178 affective state (trait anxiety), and hence performance on a judgement bias task.

179 If stereotyping birds are more pessimistic then we argue that stereotypic

180 behaviour is an indicator of poor welfare within starlings sharing the same

181 environment (and vice versa). However, any difference in cognition related to

182 stereotypic behaviour is of importance for future studies using captive birds with

183 stereotypies.

184

\section{Methods}

\section{Subjects and Husbandry}

187 The subjects were eight European starlings (four males and four females) caught

188 from the wild under license from Natural England. An equal number of juveniles

189 (birds in their first year) and adults were used. Both sex and age were

190 counterbalanced for position in the experimental laboratory and time of

191 behavioural testing. Prior to the experiment the subjects were group-housed in an

192 indoor aviary $(2.4 \times 2.15 \times 2.3 \mathrm{~m})$ with wood chippings covering the floor, dead

193 trees for perching and cover, and shallow trays of water for bathing. At the start

194 of the experiment, the birds were moved into individual cages ( $75 \mathrm{~cm}$ wide $\times 45$

$195 \mathrm{~cm}$ deep $\times 44 \mathrm{~cm}$ high) where visual and auditory contact with at least four

196 conspecifics was possible. Previous studies in solitary-housed starlings have

197 shown that differences in cage dimensions and enrichments cause changes in 
198 behaviour, condition and affective state (Bateson \& Matheson 2007; Matheson et

199 al. 2008; Asher et al. 2009), hence we were confident that the stress of individual 200 housing would not cause a ceiling effect constraint in the present study. During all 201 training phases the cages were furnished with enrichments suggested to improve 202 the welfare of captive starlings, namely: natural branches, a water bath and a 203 tray of bark chippings. The light: dark cycle was maintained at 14:10 hours. At all 204 times, other than those described below, the subjects had ad libitum access to 205 Purina kitten food, supplemented with fruit and mealworms (Tenebrio larvae). 206 Drinking water was available at all times.

207 The birds were subject to the same daily routine throughout the study: 208 cage husbandry at 8:00 a.m., followed by two hours of food deprivation to 209 increase the subjects' motivation for the learning task, followed by approximately 210 one hour of experimental trials (either learning or performing the cognitive bias 211 task - see below). On completion of the trials, the birds were once more allowed 212 to feed ad libitum. Due to the staggering of trials (four birds were tested at a 213 time), all experimental procedures were completed by approximately midday.

214 The birds' behaviour in the absence of the experimenter was recorded 215 using two video cameras. Four birds were recorded per half hour between 3:00 216 and 4:00 p.m. The order of recording was counterbalanced such that each bird 217 was recorded alternately from $3: 00-3: 30$ or $3: 30-4: 00$ on each day.

\section{Cognitive Bias Task}

219 We used a visual conditional discrimination task with differential rewards whereby 220 the birds had to attend to the colour of the background ( $\mathrm{S}+$ or $\mathrm{S}-$ ) to predict 221 which of two visually distinct dishes placed on it contained a hidden treat and 222 which was empty (see Fig. 1a). In S+ trials the treat was of a higher value than 223 in S- trials. This difference in the level of reinforcement was required to ensure 224 that active responses would be given to both the S+ and $\mathrm{S}$ - stimuli but that these 225 responses could be differentiated by the subjects' motivation to exploit the 226 reward. Once birds had learnt this discrimination the test of cognitive bias 227 involved presenting intermediate backgrounds between S+ and S- and recording 228 which of the two dishes the bird chose in extinction (see Fig. 1b). We predicted

229 that a more pessimistic bird would be more likely to interpret the ambiguous 230 background as S-, and would therefore be more likely to choose the dish 231 reinforced in S- trials. Matheson (2007) has previously piloted a version of this 232 choice task in starlings. 

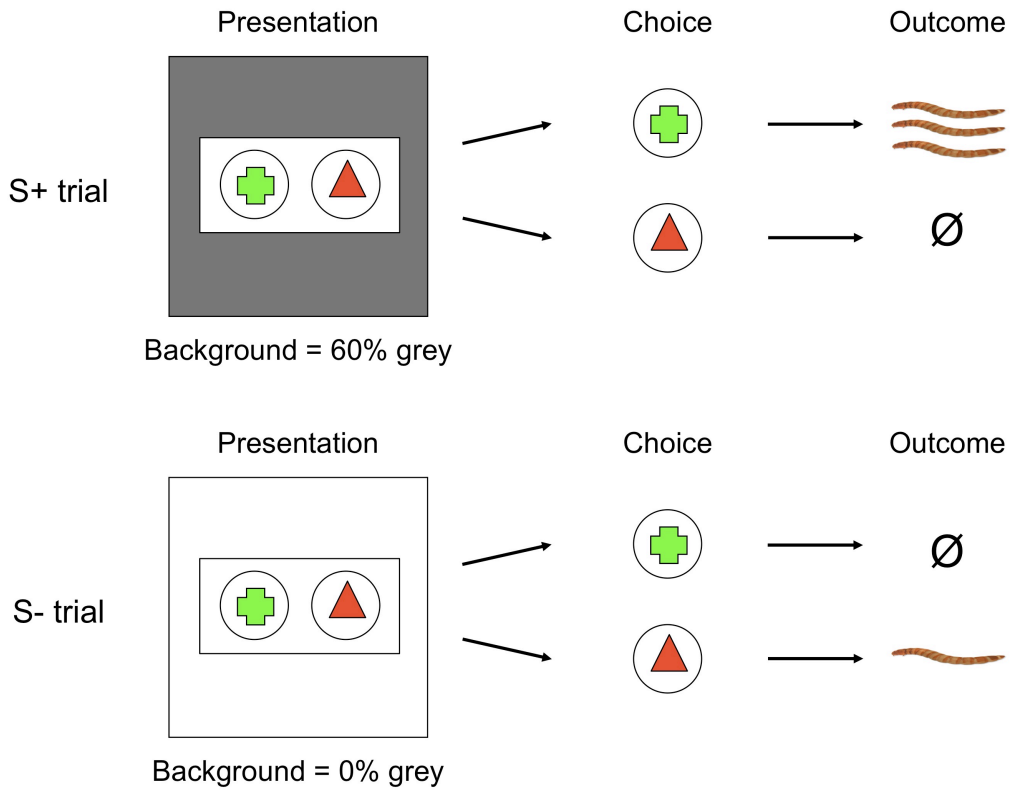

a)

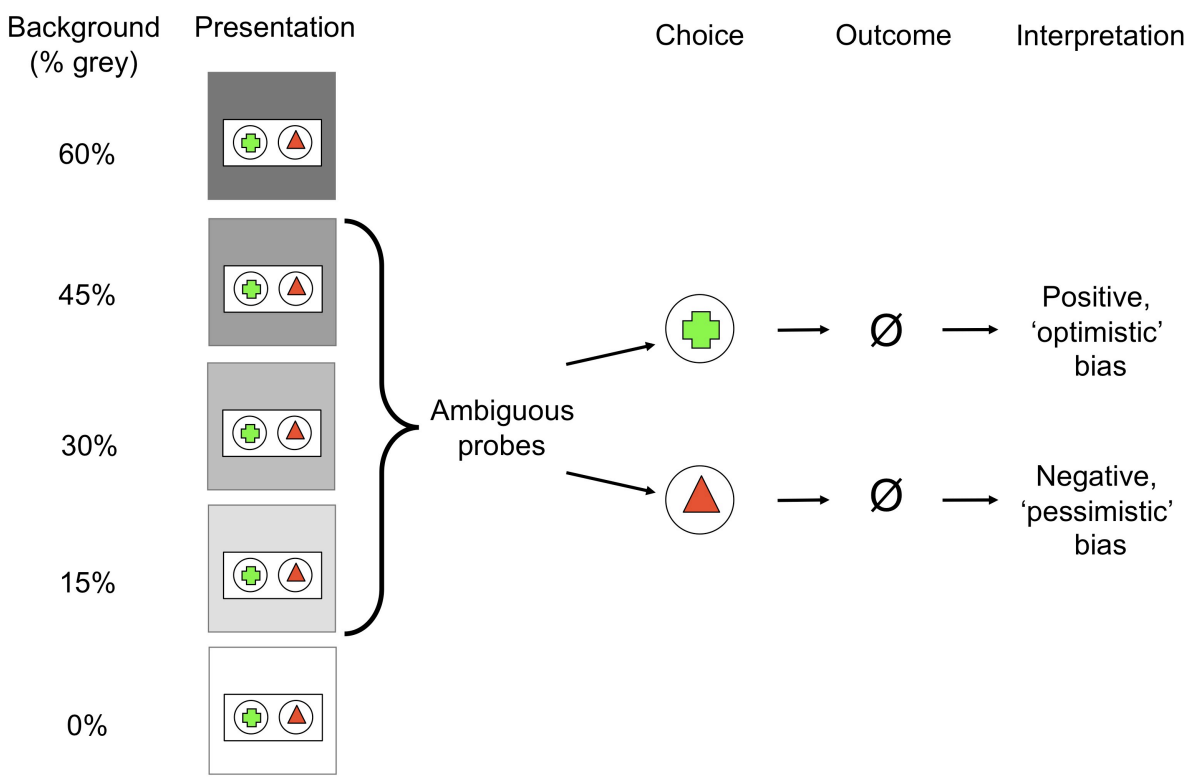

b)

Fig. 1 (a) Details of the conditional discrimination task. The reward for a correct decision in the $\mathrm{S}+$ trials was three mealworms, in the $\mathrm{S}$ - trials it was one mealworm. (b) Details of the cognitive bias test showing the three ambiguous probe background shades and our interpretation of the birds' choices. 
234 Apparatus. Two opaque Petri dishes ( $5 \mathrm{~cm}$ diameter $\times 0.5 \mathrm{~cm}$ high) were

235 mounted $3 \mathrm{~cm}$ apart on a ceramic tile $(15 \mathrm{~cm} \times 15 \mathrm{~cm})$. The background colour of

236 the tile was used as the discriminative stimulus ( $\mathrm{S}+$ or $\mathrm{S}-$ ) and was altered by

237 affixing printed paper to the tile (Fig. 1a). S+ was printed using the settings of

238 Hue $0^{\circ}$; Saturation 0\%; and Brightness $40 \%$ in Microsoft Powerpoint, and is

239 henceforth referred to as $60 \%$ grey. S- was printed using: Hue $0^{\circ}$; Saturation

$2400 \%$; and Brightness $100 \%$, and is henceforth referred to as $0 \%$ grey. The

241 intermediate stimuli used as 'probes' for cognitive bias assessment were printed

242 using the same Hue and Saturation values but varying Brightness to $55 \%, 70 \%$

243 or $85 \%$ giving shades henceforth referred to as $45 \%, 30 \%$ and $15 \%$ grey

244 respectively (Fig. 1b). All subjects were trained to associate the $60 \%$ grey

245 background (i.e. $\mathrm{S}+$ ) with a three-mealworms reward and the $0 \%$ grey

246 background (i.e. S-) with a one-mealworm reward (Matheson's (2007) data

247 showed that there was no effect of whether the higher reward occurred at the

248 dark or light end of the stimulus spectrum). Pilot experiments had established

249 that starlings in the same experimental set-up expressed a significant preference

250 for three mealworms over one mealworm, confirming our assumption that the

251 larger reward is of higher value. The Petri dishes were covered by circular

252 cardboard lids (6.5 cm diameter) printed with one of two distinct stimuli (a red

253 triangle or a green cross) that signalled which of the dishes contained the reward.

254 The stimuli on the lids were also replicated on paper circles that were glued to the

255 inside bottom of the Petri dishes (such that they were visible below the reward

256 once the lid had been removed). Pilot experiments had also established that

257 these stimuli were easily discriminable to the birds. Half of the birds were trained

258 to associate the red triangle with the 3-mealworm reward and the green cross

259 with the 1-mealworm reward whilst the other half were trained to the reverse

260 assignment.

261 Training the birds for the cognitive bias task involved three phases:

262 learning to flip lids, no-choice learning of the S+/S-conditional discrimination and

263 free-choice learning of the full task with no ambiguous intermediates.

265 Training lid-flipping. A tile with a single, centrally placed Petri dish and a plain

266 yellow cardboard lid were used in the initial lid-flipping training. The birds were

267 rewarded with a mealworm placed on the yellow lid that was in turn placed on the

268 tile. To facilitate learning of the lid-flipping task, the lid was then moved so as to

269 cover more of the Petri dish whilst the mealworm was moved to within the dish

270 (though still visible). In order, the lid was placed: on the ceramic tile, next to the

271 dish which contained the mealworm; leaning against the dish but not obscuring 
272 the contents at all; progressively obscuring more of the contents (i.e. $1 / 4$ on, $1 / 2$

273 on, $3 / 4$ on) until it covered the entire dish and the contents could only be seen and

274 recovered by moving the lid aside. Sixteen trials were given each day, each

275 lasting for $60 \mathrm{~s}$ with a 180-s inter-trial interval (ITI). The bird was required to eat

276 the mealworm within $60 \mathrm{~s}$ or the previous training stage was repeated. The bird

277 was considered to have learnt to lid-flip once it had completed 16 consecutive

278 trials with the lid fully covering the dish. This training phase continued until all

279 birds had learnt the task.

281 Training the conditional discrimination. Next, birds were given a no-choice

282 task using the two backgrounds ( $\mathrm{S}+$ and S-) and two lid-types that would be

283 used in the final experiment (red triangle and green cross). They were presented

284 with one open dish and one covered dish. The covered petri dish was always

285 represented by the correct choice given the background context (i.e. if a $60 \%$

286 grey background was shown then only the correct lid would be present on a Petri

287 dish and this would contain the 3-mealworms reward). Trials were separated into

288 blocks such that each bird had six presentations of the $60 \%$ grey background in a

289 row followed by six presentations of the $0 \%$ grey background with the order of

290 the blocks alternating between days. Each trial lasted $60 \mathrm{~s}$ with a $180-\mathrm{s}$ ITI. Upon

291 completion of the 12 trials, the birds were then given six probe trials. In these the

292 birds were presented with a choice of both lids; beneath the correct lid (given the

293 background context) was the appropriate reward. Half of the probe trials used the

$29460 \%$ grey background and half used the 0\% grey with the order pseudo-

295 randomised. The birds were considered to have learnt the discrimination when

296 they were above significance on the binomial test over the course of three days of

297 testing (14 correct choices out of 18 ). This phase continued for all birds until the

298 last subject had learnt the discrimination.

299 Training partial reinforcement. Next, free-choice trials were given, and the

300 probability of reward was gradually reduced using randomly interspersed

301 unrewarded trials. Fifteen trials were conducted per day in this phase (again with

302 a trial duration of $60 \mathrm{~s}$ and ITI of $180 \mathrm{~s}$ ). Of these trials, all were rewarded on the

303 first day, only 12 were rewarded on the second and third days, and only 10 were

304 rewarded on the fourth day. The last day corresponded exactly to the trials that

305 would be conducted as part of the cognitive bias task: 15 trials, five of which

306 would not be reinforced. Reduction in the reinforcement probability was intended

307 to prolong the conditioned response (CR) during the cognitive bias trials when

308 ambiguous probe trials would be unrewarded and hence would cause the CR to

309 extinguish. 
311 Cognitive bias trials. The experimental cognitive bias test involved one session

312 of 15 trials per day. Of these, five trials were reinforced presentations of the $60 \%$

313 grey background, five trials were reinforced presentations of the $0 \%$ grey

314 background, two trials were unreinforced presentations of the $60 \%$ and $0 \%$ grey

315 backgrounds and the remaining three were unreinforced presentations of each of

316 the intermediate, ambiguous backgrounds (15, 30 and 45\% grey). The order of

317 presentation was pseudo-random, although we avoided contiguous unrewarded

318 trials. As in the training trials, if a choice was not expressed within $60 \mathrm{~s}$ then the

319 trial was terminated and the usual ITI was observed.

320 In each phase of the experiment the choice made on every trial was

321 recorded for each subject. A choice was either recorded as appropriate for S+

322 (indicative of an optimistic bias) or appropriate for S- (indicative of a pessimistic

323 bias). In addition, the time taken from presentation of the tile until a choice was

324 expressed was also recorded (defined as when the lid was moved such that the

325 bird could observe the reward). Latency has been successfully used as a response

326 variable in previous cognitive bias studies with rats (Burman et al. 2008a), and

327 typically correlates well with choice in previous studies with starlings (e.g.

328 Bateson and Kacelnik 1995).

\section{Housing manipulations}

330 The cognitive bias trials were run daily over the course of three weeks whilst

331 environmental enrichment in the cages was varied each week in a repeated

332 measures design. For the first and third weeks the birds were in environmentally

333 enriched conditions similar to those from prior cognitive bias experiments in

334 starlings (natural wood branches; water for bathing; and a tray filled with bark

335 for natural probing opportunities: Bateson and Matheson 2007). For the second

336 week these enrichments were removed (non-enriched conditions) and the birds

337 were left with the empty water and bark containers and uniform dowel perches.

338 In order to standardise and minimise the disruption caused by the experimenter

339 physically changing the housing conditions, the birds were caught and transferred

340 to new, appropriately furnished (enriched/unenriched) cages. This was done on

341 the day before each week of cognitive bias trials began (i.e. they were transferred

342 three times at weekly intervals).

\section{Scoring stereotypic behaviour}

344 The most easily quantifiable stereotypy in caged starlings is a complete

345 backwards aerial flip (or somersault; Greenwood et al. 2004; Brilot et al. 2009a). 
346 In previous work on stereotypic behaviour from this data set, we counted the

347 number of somersaults for each bird, classifying a somersault as being any

348 movement where the bird's feet passed above its head (Brilot et al. 2009a).

349 These counts were scored, using J-Watcher v1.0 (Blumstein et al. 2000), from

350 one half-hour recording per week for each subject for the six weeks of the

351 training period prior to the cognitive bias trials. Since not all birds exhibited

352 somersaulting, we classified each as having exhibited somersaulting behaviour or

353 not. We know from a previous study using data from these subjects that

354 somersaulting behaviour is closely related to other abnormal repetitive

355 behaviours, is associated with more repetitive movement patterns and with

356 higher activity levels (Brilot et al. 2009a). Somersaulting therefore acts as a

357 useful proxy measure for generally abnormal and repetitive behaviour.

\section{Statistical Analysis}

359 All statistical analyses were carried out using SPSS 16.0 for Mac (SPSS Inc.,

360 Chicago, IL, U.S.A.). All data were modelled using repeated measures General

361 Linear Models (GLMs), with assumptions being checked and the data being

362 transformed prior to analysis where appropriate. Some of the birds developed a

363 side bias during the three weeks of cognitive bias trials; we reduced the effect of

364 the bias by discarding data from a bird for any day when it failed to reach

365 criterion (at least 10 out of 12 correct) for the subset of trials with the trained

366 backgrounds ( $0 \%$ and $60 \%$ grey). Excluded data comprised 8 out of 56 bird days

367 (4 of which were for one subject) for week 1; 9 out of 56 bird days for week 2

368 (spread across four birds); and none in the last week (one bird day comprises

369 data from one subject for one day). Since we artificially reduced the variance in

370 the response to the trained unambiguous stimuli, we excluded the data from

371 them in our analyses.

\section{$372 \quad$ Ethical note}

373 Our study adhered to the Association for the Study of Animal Behaviour's

374 Guidelines for the Use of Animals in Research and also passed internal ethical

375 review. Birds were inspected on a daily basis by the experimenter, were released

376 back into free-flight aviaries after the experiment and showed no signs of adverse

377 effects. Following completion of our studies, they received a full health inspection

378 by a qualified veterinarian prior to release at the original capture site. 


\section{Results}

\section{Cognitive Bias Task}

381 Training. The birds took $4.38 \pm 2.13$ days (mean $\pm s d$ ) to learn the lid-flipping 382 task. All subjects had learnt the task by day seven. The birds took an additional $38313.25 \pm 4.33$ (mean $\pm s d$ ) days to reach criterion on the conditional discrimination 384 task. All birds had learnt the task after 20 days of discrimination training. In the 385 last three days of discrimination training, before partial reinforcement was 386 introduced, there was a difference in the latency of the birds to make a choice 387 with $0 \%$ and $60 \%$ grey backgrounds, with birds being slower in the $0 \%$ grey 388 background trials where the reward was only one mealworm compared with $60 \%$ 389 grey background trials where the reward was three mealworms (paired t-test: $t_{7}$ $390=2.463, p=0.043$ ).

\section{Probability of choosing the stimulus associated with the larger reward.}

393 To establish whether cognitive bias was altered by our housing manipulation, we

394 compared the probability of the subjects choosing the lid stimulus associated with 395 the larger reward for the three ambiguous probe shades in each of the three 396 weeks of the test (see Fig. 2 which plots the data from all unreinforced trials 397 across all five shades, both ambiguous probes and non-ambiguous trained 398 stimuli, to allow a baseline comparison). We used probe background value and 399 week number as categorical within-subjects factors in a repeated-measures 400 ANOVA, with the probability of choosing the lid stimulus associated with the 401 larger reward as the dependent variable. There was a significant effect of the 402 probe background shade on the birds' choices $\left(F_{2,8}=58.90, p<0.001\right)$ but there 403 was no significant effect of either the week of testing $\left(F_{2,8}=0.14, p=0.871\right)$ or 404 the interaction between the week of testing and probe background shade $\left(F_{4,16}=\right.$ $4050.01, p=1.00)$. Since three of the subjects did not respond to at least one probe 406 background value for at least one of the weeks of testing, only five subjects could 407 be included in a repeated-measures ANOVA. This statistical test is therefore likely 408 to be conservative. To reduce the likelihood of a type II error, we re-ran the 409 analysis using the data from each probe background value in turn and included 410 week as the only independent variable. This revealed that week of testing still 411 had no significant effect on the choices expressed (Probe $15 \%$ grey background: $412 \quad F_{2,12}=0.24, p=0.792 ;$ Probe $30 \%$ grey background: $F_{2,8}=0.02, p=0.982 ;$ 413 Probe $45 \%$ grey background: $F_{2,14}=1.01, p=0.390$; changes in the degrees of 414 freedom represent changes in sample size since some subjects failed to give a 415 response to the probe in a given week). 


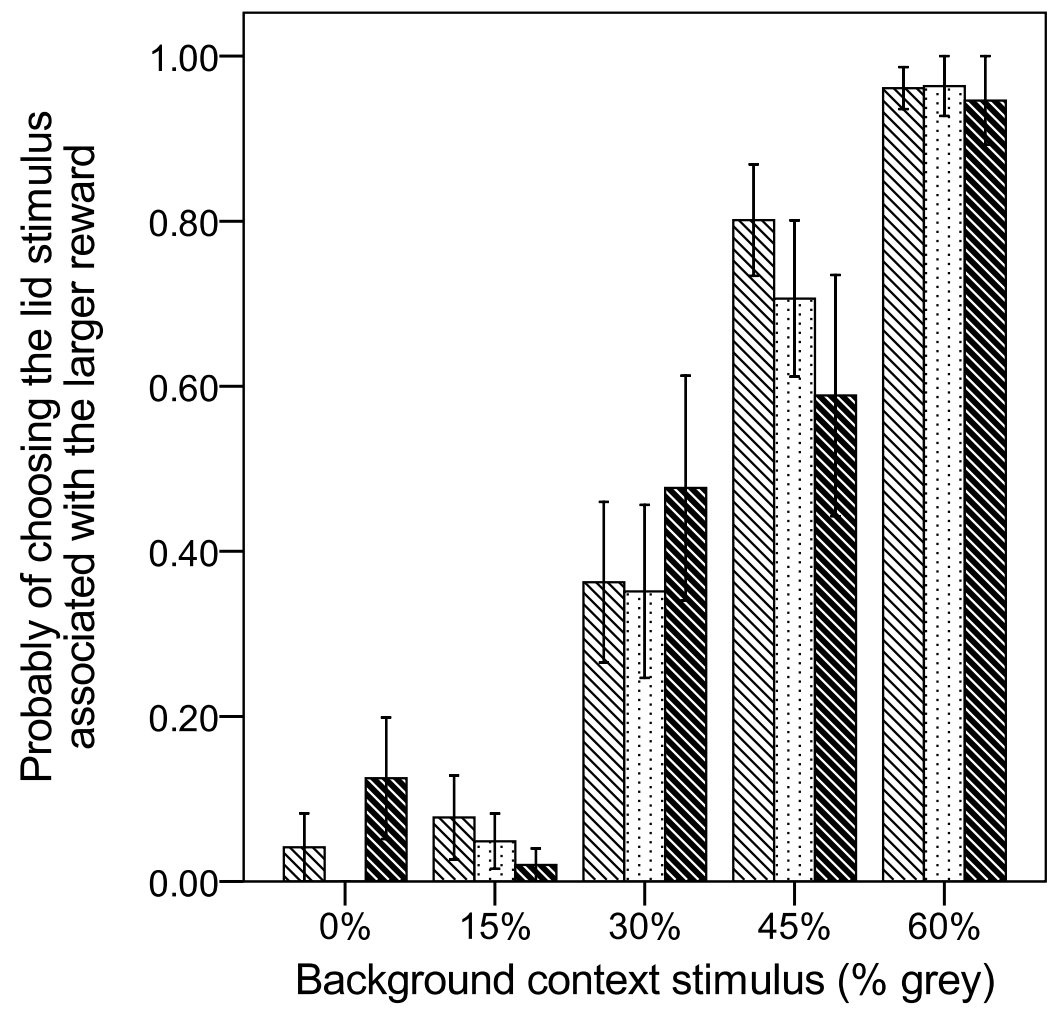

Fig. 2 Probability of choosing the stimulus associated with the higher reward during cognitive bias trials averaged across all subjects. Percentage grey values signify which background context was presented. Light hatched bars represent choices during week 1 (enriched conditions); dotted bars represent choices during week 2 (unenriched conditions); dark hatched bars represent choices during week 3 (enriched conditions). Bars show the mean for the 8 birds \pm one standard error. 
417 Latency to choose. Although the choice data showed no effect of our housing

418 manipulation, there remained the possibility that the birds' expectancy of reward

419 size in the ambiguous probe trials was reflected in their latencies to respond.

420 Where a bird failed to exhibit a choice within the time allowed, it was allocated

421 the maximum trial duration of $60 \mathrm{~s}$. We calculated the latency to flip a lid for each

422 probe stimulus relative to the 3 -mealworm reinforced stimulus (60\% grey; Fig.

423 3). We predicted an increase in latency in non-enriched conditions (reflecting

424 pessimism regarding the expected reward), followed by a decrease in latency on

425 return to enriched conditions (reflecting recovered optimism). However,

426 inspection of Fig. 3 suggests that if anything, latencies in the ambiguous probe

427 trials increased across the three weeks of testing. A repeated-measures ANOVA

428 (with week number and probe background as within-subjects fixed factors)

429 showed that latencies differed significantly across the probe background value

430 and across the three weeks of trials (Probe value: $F_{2,14}=10.22 p=0.002$; Week

431 number: $F_{2,14}=7.92 p=0.005$ ). Post-hoc analysis using t-tests (with a

432 Bonferroni correction applied) revealed a significant difference in the latency to

433 respond to the $30 \%$ and $45 \%$ grey background probes $(p=0.001)$, but all other

434 pairwise comparisons were non-significant ( $p>0.18$ for all). Similarly,

435 Bonferroni-corrected post-hoc analysis revealed a significant difference in the

436 latency to respond for weeks 1 and $3(p=0.039)$ but all other pairwise

437 comparisons between weeks were non-significant ( $p>0.15$ for all). There was no

438 significant interaction effect of the probe background value and week number on

439 the latency to choose (Mauchly's test revealed that the assumption of sphericity

440 was not tenable $\left(X_{9}=32.48, p<0.001\right)$, therefore the Greenhouse-Geisser

441 correction was applied: $\left.F_{1.94,13.55}=0.64, p=0.537\right)$.

443 Cognitive Bias Task and Individual Behavioural Differences

444 To ascertain whether presence of stereotypic behaviour (as an indicator of

445 affective state) predicts the probability of choosing the stimulus associated with

446 the larger reward we conducted a repeated-measures ANOVA with probe

447 background value as a within-subjects factor and the presence or not of

448 somersaulting behaviour as a between-subjects factor. We used only the data

449 from the first week of trials for this analysis to minimise the effects of learning

450 observed in the second and third weeks and to avoid any potential confound from

451 the housing manipulation. The data on somersaulting showed that only three out

452 of eight subjects demonstrated somersaulting behaviour during the period prior 


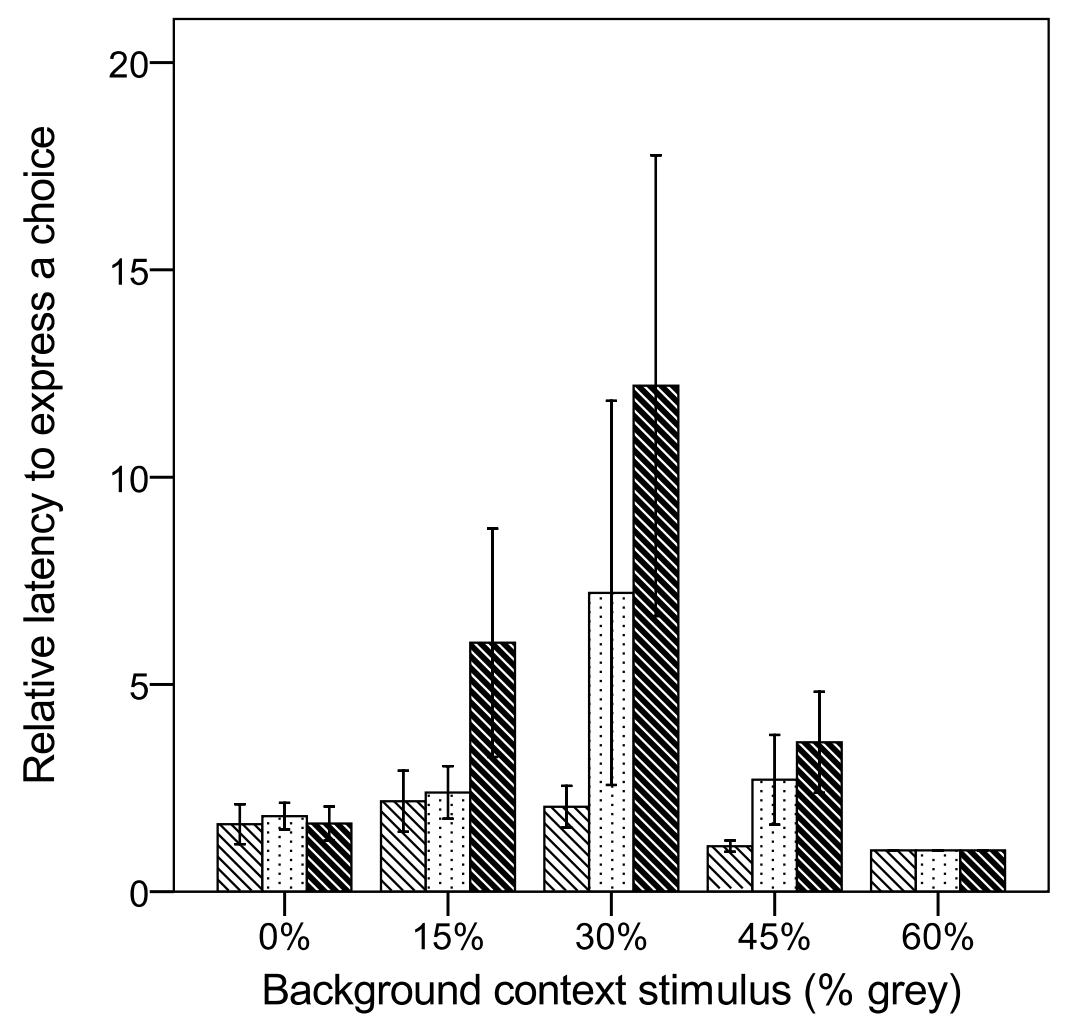

Fig. 3 Latency to approach and flip the lid averaged for each background context stimulus. The latency is corrected for each individual bird by dividing the actual mean latency by the mean latency to flip the lid of the rewarded "threemealworms" stimulus (i.e. the $60 \%$ grey background trials) during the same week of trials. The percentage grey values signify which background context was presented. Light hatched bars represent latencies during week 1 (enriched conditions); dotted bars represent latencies during week 2 (unenriched conditions); dark hatched bars represent latencies during week 3 (enriched conditions). Bars show the mean for the 8 birds \pm one standard error. 
453 to the cognitive bias trials (see Brilot et al. 2009a). None of the subjects showed

454 somersaulting behaviour during the three-week cognitive bias trial period. The

455 analysis showed that there was a significant effect of the probe background value

456 on the stimulus chosen $\left(F_{2,12}=32.33, p<0.001\right)$. Post-hoc analysis (with

457 Bonferroni corrections applied) revealed that there was a significant difference in

458 the response to the $15 \%$ vs. $45 \%$ grey backgrounds ( $p=0.002$ ) and the $30 \%$ vs.

$45945 \%$ grey backgrounds $(p=0.001)$ but there was no significant difference in the

460 response to the $15 \%$ vs. $30 \%$ grey backgrounds ( $p=0.149)$. Somersaulting

461 behaviour had an effect on the choices made, manifested as a significant

462 interaction between probe background value and somersaulting $\left(F_{2,12}=4.40, p=\right.$

4630.037 ; Fig. 4), though there was no significant main effect of somersaulting $\left(F_{1,7}\right.$

$464=1.56, p=0.259)$. To establish the meaning of this interaction, we conducted

465 repeated contrasts which revealed a significant interaction when comparing the

466 choices made by stereotyping and non-stereotyping individuals in response to the

$46715 \%$ background probe vs. the $30 \%$ background probe $\left(F_{1,6}=6.36, p=0.045\right)$

468 and the $30 \%$ vs. $45 \%$ background probe $\left(F_{1,6}=11.54, p=0.015\right)$. Examination

469 of figure 4 confirms this interaction: somersaulting birds were more likely to

470 choose the stimulus associated with the lower reward value, but this difference

471 was only expressed in response to the $30 \%$ grey background probe.

\section{Discussion}

476 In this paper we set out first, to develop an improved cognitive bias task for

477 starlings, and, second, to extend previous work in animals by asking whether

478 cognitive biases are correlated with individual differences in the incidence of

479 abnormal behaviour. Although we succeeded in training birds on our novel

480 cognitive bias task, we failed to find the predicted changes in cognitive bias,

481 expected with changes in housing conditions. However, we did find that

482 performance on the task was predicted by individual differences in whether or not

483 birds showed stereotypic somersaulting behaviour. Below we discuss the

484 explanations for these findings and their implications in the context of our original 485 aims. 


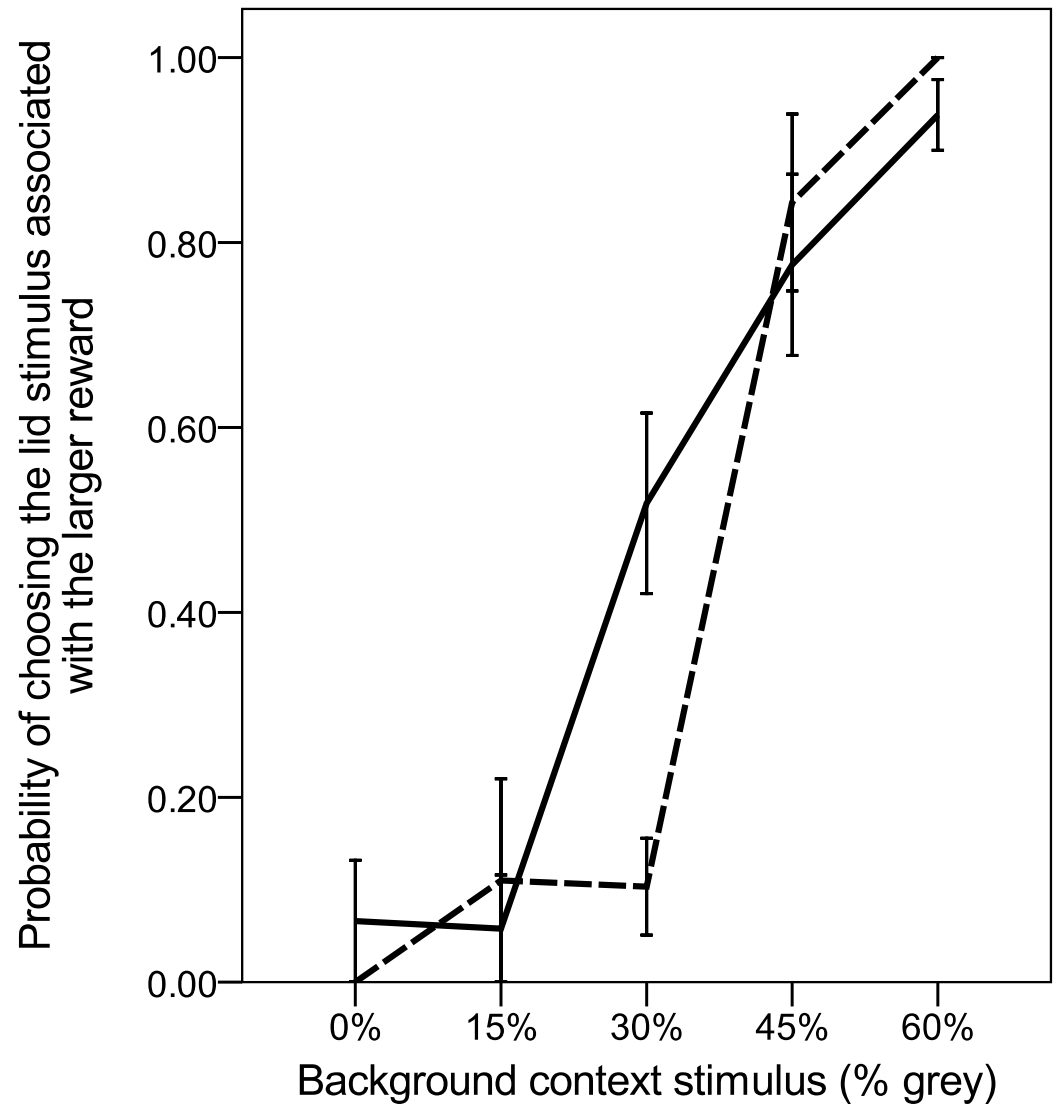

Fig. 4 Probability of choosing the stimulus associated with the larger reward for each background context stimulus in the first week of cognitive bias trials (enriched conditions). The subjects are divided into those that exhibited somersaulting behaviour at some stage during the first six weeks of the training period (dashed line) and those that did not (solid black line). Data points show the mean for the 8 birds \pm one standard error. 


\section{Cognitive bias tasks and learning}

487 The birds' judgment biases, as measured by their choice of which lid to flip during

488 ambiguous probe trials, were not affected in any consistent manner by our

489 manipulation of their housing conditions (Fig. 2). The cognitive bias task

490 therefore failed to detect any changes in affective state that might have been

491 induced by the change in environmental conditions. However, this is unsurprising

492 given the additional data on the increase in latencies to choose across the three

493 weeks of cognitive bias testing (Fig. 3). This increase is inconsistent with a

494 cognitive bias interpretation, and instead suggests that the birds were learning

495 that their choice in ambiguous probe trials was never rewarded with mealworms.

496 Indeed, by the third week of the testing, two birds completely failed to make a

497 choice in the $30 \%$ grey background probe trials. We therefore conclude that the

498 birds learnt quickly that the intermediate probe stimuli were never associated

499 with reinforcement, thus rendering the probe trials unambiguous by the second

500 week of testing, and the task ineffective for detecting changes in affective state.

501 Ours is the first cognitive bias experiment to find evidence for such rapid learning

502 and loss of ambiguity in probe trials raising the question of why this occurred.

503 The experiment presented is the only cognitive bias task, that we are

504 aware of, that has employed this specific repeated measures methodology (i.e.

505 from condition $A$ to condition $B$ and return to condition $A$ ). The rationale that this

506 allowed each bird to be its own control was justified given the large range of

507 inter-individual variability we found in response to the ambiguous probes in the

508 initial stages. A between-groups design would have required greater sample sizes

509 to detect similar effects given this noise from individual differences. However, the

510 repeated measures design also meant that learning became a significant factor in

511 reducing the sensitivity of the cognitive bias measure. The cognitive bias testing

512 lasted 21 days with the birds having 21 exposures to each probe stimulus over

513 this time. In the two previous cognitive bias experiments on starlings (Bateson

514 and Matheson 2007; Matheson et al. 2008), the test phases lasted for 10 and 20

515 days respectively and the birds had 36 and up to 80 exposures to each probe

516 stimulus over this time respectively. It is not possible to compare the latter study

517 with the current study since the stimuli used were entirely different. However, the

518 former study (Bateson and Matheson 2007) used similar stimuli and training

519 techniques to the current experiment. In fact, the stimuli used in the current

520 study were actually drawn from a smaller range than in Bateson and Matheson

521 (2007) and therefore we would have predicted that if anything, the ambiguous

522 stimuli would have been harder to distinguish from the trained S+ and S- stimuli,

523 not easier. 
In an attempt to resolve this apparent contradiction we re-examined the data presented in Bateson and Matheson (2007) to investigate whether it could be re-interpreted as the result of learning as opposed to a change in cognitive bias. If the birds learnt that the ambiguous stimuli were never reinforced, this would have resulted in a reduced probability of lid flipping in the second treatment received by the birds, and hence behaviour interpreted as indicating a more pessimistic cognitive bias in the second treatment. In fact, this is exactly what was observed. Fig. 2 of Bateson and Matheson (2007) shows a reduced probability of lid-flipping when the birds moved from enriched to standard conditions. This was interpreted as a cognitive bias shift, since birds in a more negative affective state would be more likely to negatively interpret the stimulus and therefore avoid the lids. However, in the same figure, the birds that received the treatments in the reverse order (i.e. standard to enriched) also showed a (non-significant) reduction in lid-flipping in their second treatment. Taken together with the evidence from the current study showing the same trend, these data strongly suggest that the birds in Bateson and Matheson (2007) were learning that the ambiguous probes were never reinforced as opposed to exhibiting a change in cognitive bias.

The possibility of subjects learning rapidly that ambiguous probe trials are unreinforced is therefore a difficulty for experiments designed to detect changes in cognitive bias. The most successful cognitive bias experiments have most likely circumvented this problem by using a between-subjects design with a short duration of testing with ambiguous probes (Harding et al. 2004; Burman et al.

547 2008a). However, even with these designs, the possibility remains that

548 reductions in the probability of responding or latency to respond, interpreted as more pessimistic judgment biases, could actually be attributable to effects of

550 stress on speed of learning. Though the general validity of the Yerkes-Dodson law

551 (that there is an inverted U-response function linking stress and learning speed)

552 is questioned, there is confirmatory evidence linking mild levels of stress and

553 improved memory formation (Mendl 1999). For instance, there is evidence in rats

554 that pharmacologically-induced mild stress (administration of low doses of

555 corticosterone) can enhance learning (Okuda et al. 2004), but only under

556 conditions of emotional arousal (in this case response to a novel object). It is

557 therefore a possibility that experiments aimed at assessing a cognitive bias may

558 be confounded by an additional interaction between stress and learning (as well

559 as stress and cognitive interpretations). In short, individuals under mildly

560 stressful conditions may learn more quickly that ambiguous probes are

561 unreinforced and therefore show a reduced response in both go/go and go/no-go 
562 experimental designs. A potential solution to this difficulty lies in the use of

563 paradigms that require only a single exposure to ambiguous, unreinforced probe

564 stimuli where learning cannot be a confound (see Brilot et al. 2009b for a first

565 attempt at such a task).

\section{Cognitive bias and individual behavioural differences}

567 The results from the first week of cognitive bias testing suggest that performance

568 is predicted by whether starlings display stereotypic behaviour in the form of

569 somersaulting. Individuals that performed somersaults demonstrated a

570 significantly more pessimistic interpretation of the most ambiguous ( $30 \%$ grey)

571 probe stimulus than non-stereotyping individuals. Though there proved to be no

572 relationship between responses to the $15 \%$ and $45 \%$ grey probes and

573 somersaulting behaviour, this is unsurprising given the reduced ambiguity of

574 these probes as compared to the 30\% grey background. Fig. 3 shows that these

575 two probes were treated as approximately equivalent to the trained $\mathrm{S}+$ and $\mathrm{S}-$

576 backgrounds as judged by the birds' choice responses. Any sensitivity to

577 individual differences in response was therefore likely overshadowed by a

578 generalised, strong conditioned response to the previously-encountered stimuli.

579 This study examined individual differences in somersaulting behaviour and

580 the relationship between this stereotypy and cognitive bias. Elsewhere we have

581 analysed data on behaviour patterns in the learning phase of the current

582 experiment (Brilot et al. 2009a). This showed that repetitiveness of movement

583 patterns, abnormal stereotypic behaviour (including somersaulting), and the use

584 of abnormal perching locations are all positively correlated in a complex that is

585 suggestive of a behavioural response to caging. Additionally, it is known that an

586 increase in the repetitiveness of behaviour is correlated with the housing

587 conditions of starlings (both with cage type and enrichments: Asher 2007; Asher

588 et al. 2009). There is some evidence to suggest that this may be related to a

589 thwarted escape response, as originally suggested by Maddocks et al. (2002).

590 Our findings here are therefore suggestive that performance on the cognitive bias

591 task, and by implication affective state, relates to this suite of abnormal and

592 repetitive behaviour measures. As outlined in the introduction, it is generally

593 considered that the presence of stereotypic behaviour indicates poor welfare

594 when comparing differing housing regimes. However, the evidence for animals

595 that share the same captive conditions is equivocal, with the majority of studies

596 suggesting that stereotyping individuals actually display indicators of better

597 welfare than non-stereotyping individuals (Mason and Latham 2004; Mason

598 2006). The present study suggests that the presence of stereotypic behaviour in 
599 starlings is an indicator of poor welfare, even when comparing individuals who

600 share the same housing conditions.

601 There are a number of reasons why stereotypic behaviour might be an

602 indicator of negative affective state and therefore of poor welfare (Mason and

603 Latham 2004). We suggest that the typical starling stereotypy, somersaulting,

604 observed in our study, fulfils the criteria in Table 2 of Mason and Latham (2004)

605 for a stereotypic behaviour that is an index of poor welfare (specifically an index

606 of frustration: Table 3, Mason \& Latham 2004). First, the stereotypy is not a

607 suitable replacement for the natural activity. Since we hypothesise that the

608 behaviour patterns and stereotypic behaviour are indicators of a thwarted escape

609 response, there is no likelihood that they act as a suitable substitute. Second, it

610 seems unlikely that this behaviour has a 'mantra effect', i.e. a positively

611 reinforcing ability to reduce stress, though the present data do not allow us to

612 exclude this possibility. Third, stereotypic behaviour in our study was embedded

613 within a suite of flexible behaviours. The individuals that demonstrated

614 somersaulting behaviour were still able to attend to and complete all training

615 tasks. There was no negative relationship between stereotypic behaviour and the

616 length of training across the subjects as might be expected if stereotyping

617 individuals were unwilling or unable to attend to external stimuli. Fourth and

618 finally, stereotypic behaviour seems to have been elicited 'appropriately' within

619 the context of an escape response. Somersaulting behaviour was expressed most

620 prominently during the first three weeks of captivity (Brilot et al. 2009a) and

621 subsequently decreased. However, though somersaulting decreased over time

622 during the experimental video recordings (when no humans were present), it was

623 still stimulated to an extent by the presence of the experimenter during daily

624 cognitive bias training and husbandry (personal observations). This suggests that

625 the thwarted escape response was heightened by the presence of a perceived

626 threat and therefore stereotypic behaviour was manifested. Given that the

627 stereotypic somersaulting behaviour of starlings fits the criteria for a good

628 indicator of poor welfare, we suggest that the present study indicates that

629 starlings that display more repetitive behaviour patterns and stereotypic

630 behaviours, are also suffering from a more negative affective state (as measured

631 by the cognitive bias task).

632 In conclusion, our study has revealed that rapid learning of non-reinforced

633 ambiguous probe stimuli can be a problem in cognitive bias tasks. Subjects

634 learning that ambiguous probe trials are never reinforced not only precluded us

635 from detecting changes in affective state with changes in housing conditions in

636 the current experiment, but may also have implications for other studies 
637 attempting to establish a cognitive bias where the test phase is not sufficiently 638 short. Performance on the cognitive bias task did, however, reflect behaviour in

639 captivity with regards to the incidence of abnormal repetitive behaviour (namely

640 the somersaulting stereotypy). We suggest that the wider suite of behavioural

641 traits related to repetitive behaviour is indicative of a stress response in captive

642 starlings that also reflects a more negative affective state. The cognitive bias

643 methodology therefore has merit in revealing individual differences in affective

644 state.

645

\section{Acknowledgements}

647 We thank Michelle Waddle for technical help. We thank Jim Clapp, Domhnall

648 Jennings, Stephanie Matheson, Mike Mendl, Jeroen Minderman, Liz Paul and

649 several anonymous referees for help and advice. This work was supported by two

650 grants awarded to MB from the UK's Biotechnology and Biological Sciences

651 Research Council (BB/E012000/1 and BB/05623/1).

652

653 


\section{References}

655

656

657

658

659

660

661

662

663

664

665

666

667

668

669

670

671

672

673

674

675

676

677

678

679

680

681

682

683

684

685

686

687

688

689

690

691

692

693

694

695

696

697

698

699

700

701

Asher L (2007) Welfare of captive European starlings (Sturnus vulgaris). Ph.D. Thesis. Institute of Neuroscience, Newcastle University, Newcastle upon Tyne Asher L, Davies GTO, Bertenshaw CE, Cox MAA, Bateson M (2009) The effects of cage volume and cage shape on the condition and behaviour of captive European starlings (Sturnus vulgaris). Appl Anim Behav Sci 116:286-294

Bar-Haim Y, Lamy D, Pergamin L, Bakermans-Kranenburg MJ, van Ijendoorn MH (2007) Threat-related attentional bias in anxious and nonanxious individuals: A meta-analytic study. Psychological Bulletin 133:1-24

Bateson M, Kacelnik A (1995) Preferences for fixed and variables food sources variability in amount and delay. J Exp Anal Behav 63:313-329

Bateson M, Matheson SM (2007) Performance on a categorisation task suggests that removal of environmental enrichment induces 'pessimism' in captive European starlings (Sturnus vulgaris). Anim Welfare 16:33-36

Bergvall UA, Rautio P, Luotola T, Leimar O (2007) A test of simultaneous and successive negative contrast in fallow deer foraging behaviour. Anim Behav 74:395-402

Blumstein DT, Evans CS, Daniel JC (2000) JWatcher 0.9. An introductory user's guide.

Brilot BO, Asher L, Feenders G, Bateson M (2009a) Quantification of abnormal repetitive behavior in captive European starlings (Sturnus vulgaris). Behav Process 82:256-264

Brilot BO, Normandale CL, Parkin A, Bateson M (2009b) Can we use starlings' aversion to eyespots as the basis for a novel 'cognitive bias' task? Appl Anim Behav Sci 118:182-190

Burman OHP, Parker R, Paul ES, Mendl M (2008a) A spatial judgement task to determine background emotional state in laboratory rats, Rattus norvegicus. Anim Behav 76:801-809

Burman OHP, Parker RMA, Paul ES, Mendl M (2008b) Sensitivity to reward loss as an indicator of animal emotion and welfare. Biol Lett 4:330-333

Enkel T, Gholizadeh D, von Bohlen Und Halbach O, Sanchis-Segura C, Hurlemann R, Spanagel R, Gass P, Vollmayr B (2009) Ambiguous-cue interpretation is biased under stress- and depression-like states in rats. Neuropsychopharmacol. Advance online publication. doi:10.1038/npp.2009.204

Eysenck MW, Mogg K, May J, Richards A, Mathews A (1991) Bias in interpretation of ambiguous sentences related to threat in anxiety. Journal of Abnormal Psychology 100:144-150

Flaherty CF (1996) Incentive relativity. Cambridge University Press, Cambridge

Greenwood VJ, Smith EL, Goldsmith AR, Cuthill IC, Crisp LH, Walter-Swan MB, Bennett ATD (2004) Does the flicker frequency of fluorescent lighting affect the welfare of captive European starlings? Appl Anim Behav Sci 86:145-159

Harding EJ, Paul ES, Mendl M (2004) Animal behavior: Cognitive bias and affective state. Nature 427:312-312

Maddocks SA, Goldsmith AR, Cuthill IC (2002) Behavioural and physiological effects of absence of ultraviolet wavelengths on European starlings Sturnus vulgaris. J Avian Biol 33:103-106

Mason G (2006) Stereotypic behaviour in captive animals: fundamentals and implications for welfare and beyond. In: Mason G, Rushen J (eds) Stereotypic 
animal behaviour: fundamentals and applications to welfare, Second edition edn. CABI, Wallingford, pp 325-356

Mason G, Rushen J (2006) Stereotypic animal behaviour: fundamentals and applications to welfare. In, Second edition edn. CABI, Wallingford

Mason GJ, Latham NR (2004) Can't stop, won't stop: is stereotypy a reliable animal welfare indicator? Anim Welfare 13:S57-69

Matheson SM (2007) Using cognitive bias to assess welfare in captive European starlings (Sturnus vulgaris). M.Sc. Thesis. Institute of Neuroscience, Newcastle University, Newcastle upon Tyne

Matheson SM, Asher L, Bateson M (2008) Larger, enriched cages are associated with 'optimistic' response biases in captive European starlings (Sturnus vulgaris). Appl Anim Behav Sci 109:374-383

Mendl M (1999) Performing under pressure: stress and cognitive function. Appl Anim Behav Sci 65:221-244

Mendl M, Burman OHP, Parker RMA, Paul ES (2009) Cognitive bias as an indicator of animal emotion and welfare: Emerging evidence and underlying mechanisms. Appl Anim Behav Sci 118:161-181

Okuda S, Roozendaal B, McGaugh JL (2004) Glucocorticoid effects on object recognition memory require training-associated emotional arousal. Proceedings of the National Academy of Sciences of the United States of America 101:853-858

Paul ES, Harding EJ, Mendl M (2005) Measuring emotional processes in animals: the utility of a cognitive approach. Neuroscience and Biobehavioral Reviews 29:469-491

Rich EL, Romero LM (2005) Exposure to chronic stress downregulates corticosterone responses to acute stressors. American Journal of Physiology-Regulatory Integrative and Comparative Physiology 288:R1628-R1636

Williams JMG, Watts FN, MacLeod C, Matthews A (1997) Cognitive Psychology and Emotional Disorders. John Wiley \& Sons, Chichester

Young RJ (2003) Environmental enrichment for captive animals. Blackwell Publishing, Oxford 


\section{Figure captions}

737 Fig. 1 (a) Details of the conditional discrimination task. The reward for a correct

738 decision in the $\mathrm{S}+$ trials was three mealworms, in the $\mathrm{S}$ - trials it was one

739 mealworm. (b) Details of the cognitive bias test showing the three ambiguous

740 probe background shades and our interpretation of the birds' choices.

741 Fig. 2 Probability of choosing the stimulus associated with the higher reward

742 during cognitive bias trials averaged across all subjects. Percentage grey values

743 signify which background context was presented. Light hatched bars represent

744 choices during week 1 (enriched conditions); dotted bars represent choices during

745 week 2 (unenriched conditions); dark hatched bars represent choices during week

7463 (enriched conditions). Bars show the mean for the 8 birds \pm one standard error.

747 Fig. 3 Latency to approach and flip the lid averaged for each background context

748 stimulus. The latency is corrected for each individual bird by dividing the actual

749 mean latency by the mean latency to flip the lid of the rewarded "three-

750 mealworms" stimulus (i.e. the $60 \%$ grey background trials) during the same

751 week of trials. The percentage grey values signify which background context was

752 presented. Light hatched bars represent latencies during week 1 (enriched

753 conditions); dotted bars represent latencies during week 2 (unenriched

754 conditions); dark hatched bars represent latencies during week 3 (enriched

755 conditions). Bars show the mean for the 8 birds \pm one standard error.

756 Fig. 4 Probability of choosing the stimulus associated with the larger reward for

757 each background context stimulus in the first week of cognitive bias trials

758 (enriched conditions). The subjects are divided into those that exhibited

759 somersaulting behaviour at some stage during the first six weeks of the training

760 period (dashed line) and those that did not (solid black line). Data points show

761 the mean for the 8 birds \pm one standard error. 